\title{
KIỂM ĐỊNH VÀ ĐÁNH GIÁ THANG ĐO THÁI ĐỘ ĐỊNH HƯỚNG KHỞI NGHIỆP TẠI VIẸTT NAM
}

\author{
TRÀ̀N QUANG LONG ${ }^{1}$, PHAN NHƯ MINH $^{2}$, TRÀ̀N HẢI BẰNG ${ }^{3}$ \\ ${ }^{1}$ Truò̀ng Đại học Kinh tế-Luạt \\ ${ }^{2}$ Trường Đại học Kinh tế TP. HCM \\ ${ }^{3}$ Truờng Cao Đẳng Kinh tế Đối ngoại \\ longtq@uel.edu.vn,minhpn@ueh.edu.vn,thbang@ktdn.edu.vn
}

\begin{abstract}
In light of Entrepreneurship literate, the Entrepreneurial Attitude Orientation (EAO) scale is a multidimensional self-report measure of attitudes toward entrepreneurship. There are little studies have tested the validity and reliability of the EAO scale in different social and cultural situations. This research's purpose is to examine the generalizability of the EAO scale in Vietnam context by applying two step of the preliminary research and the main research. In the former, we applied both of the qualitative and quantitative method with the finding of four dimensions scale and 20 items were removed. The latter used the Cronbach alpha for testing the scale's reliability and EFA method for examining the scale's validity. The result shows that EAO scale in Vietnam are include five dimension instead of four, with the appearance of the new one, namely ACHPC. Furthermore, 26 of the original 75 items were removed due to unsatisfactory loading. The findings generally support the generalizability of the four-dimensional model of the EAO and contribute the specialize scale in Vietnam context.
\end{abstract}

Keywords. Ý định khởi nghiệp, thái độ khởi nghiệp, EAO.

\section{GIỚI THIẸU}

Trong hệ thống lý thuyết, có hai mô hình thường xuyên được sử dụng để đo lường ý định khởi nghiệp là lý thuyết về hành vi dự định (Theory of Planned Behavior-TPB) của Ajzen và mô hình sự kiện khởi nghiệp của Shapero và Sokol (Entreprenuerial Event Model-EEM). Tuy nhiên, nhiều nhà nghiên cứ cho rằng cả hai mô hình vẫn tồn tại một số bất cập: thứ nhất, trong mô hình $\mathrm{TPB}$, Ajzen vẫn giữ quan điểm thái độ là một cấu trúc đơn và được biểu hiện qua phản ứng tình cảm đơn lập (Ajzen, 1991). Ngoài ra, TPB là mô hình ban đầu được xây dựng để dự đoán một hành vi nói chung trong tâm lý học và sau đó được áp dụng vào nghiên cứu ý định kinh doanh, trong khi ý định kinh doanh là một lĩnh vực đặc thù, chỉ diễn ra khi ý định đã được cân nhắc kỹ lưỡng, nghiêm túc. Hai là, trong mô hình EEM, ban đầu Shapero và Sokol đã không xem như một mô hình nghiên cứu ý định khi giới thiệu nó vào năm 1982, mặc dù sau đó nó đã được phát hiện và sử dụng trong nghiên cứu ý định khởi nghiệp sau này (Kermit, 2008). Mục tiêu của mô hình là chỉ để cung cấp một lời giải thích cho tiến trình dẫn đến một sự kiện kinh doanh, đó là, thời điểm tạo ra một doanh nghiệp mới (Kollmann \& Kuckertz, 2006); "Mô hình sự kiện kinh doanh là tập trung về vấn đề tạo ra doanh nghiệp mới và không phải về sự diễn tiến hướng tới việc thông qua một hành vi kinh doanh nói chung "(Fayolle et $\mathrm{al}, 2006)$. Hơn nữa, mặc dù biến "thiên hướng hành động" trong mô hình các sự kiện kinh doanh (EEM) được biết đến như là biến giải thích tại sao một người có năng lực và hoài bão trở thành một doanh nhân (và sẽ không bao giờ là một cá nhân thiếu thiên hướng hành động trước một hành vi). Tuy nhiên, Shapero và Sokol đã không đề cập đến biến này một cách rõ ràng trong mô hình ban đầu của họ.

Nhiều nhà nghiên cứu (như Abelson, 1982; Chaiken \& Stangor, 1987; Rosenberg \& Hovland, 1960) cho rằng "thái độ được xem là tương đối ít ổn định hơn những đặc điểm cá nhân, có thể thay đổi theo thời gian và qua quá trình tương tác với môi trường". Robinson đã xây dựng mô hình $\mathrm{EAO}$ để đo lường thái độ khởi nghiệp, sau một quá trình tổng quan và đánh giá có hệ thống về lý thuyết tâm lý học kết hợp với nghiên cứu về các đặc tính tiêu biểu của các doanh nhân. Mô hình EAO (Entreprenuerial Attitude Orientation) gồm 4 thang đo thành phần với 75 câu hỏi. Bốn thang đo của EAO là: 
(1)Nhu cầu thành tựu $(\mathrm{ACH})$ đề cập đến kết quả cụ thể liên quan đến việc khởi động và phát triển của một doanh nghiệp; (2) Sáng tạo (INN) liên quan đến nhận thức và hành động dựa trên các hoạt động kinh doanh theo những cách thức mới và độc đáo; (3) Cảm nhận sự điều khiển cá nhân (PC) liên quan đến nhận thức của cá nhân trong việc kiểm soát và ảnh hưởng đến việc kinh doanh của mình; (4) Cảm nhận sự tự tin (SE) liên quan đến sự tự tin và nhận thức năng lực của một cá nhân đến việc công việc kinh doanh của mình" (Robinson, Stimpson, Huefner \& Hunt, 1991).

Mỗi trong số đó bao gồm ba thành phần thái độ: cảm xúc, nhận thức và ý chí. Trong đó, cảm xúc (Affection) bao gồm cảm giác tích cực hay tiêu cực đến một vấn đề; nhận thức (Cognation) gồm niềm tin và suy nghĩ về đối tượng của thái độ; ý chí (Conative) còn gọi là thành phần hành vi - behaviour) bao gồm ý định hành vi và xu hướng. Theo Robinson, "giá trị mỗi thành phần thang đo càng lớn, khả năng dự đoán cá nhân kinh doanh càng cao" (Huefner, Hunt, và Robinson, 1996).

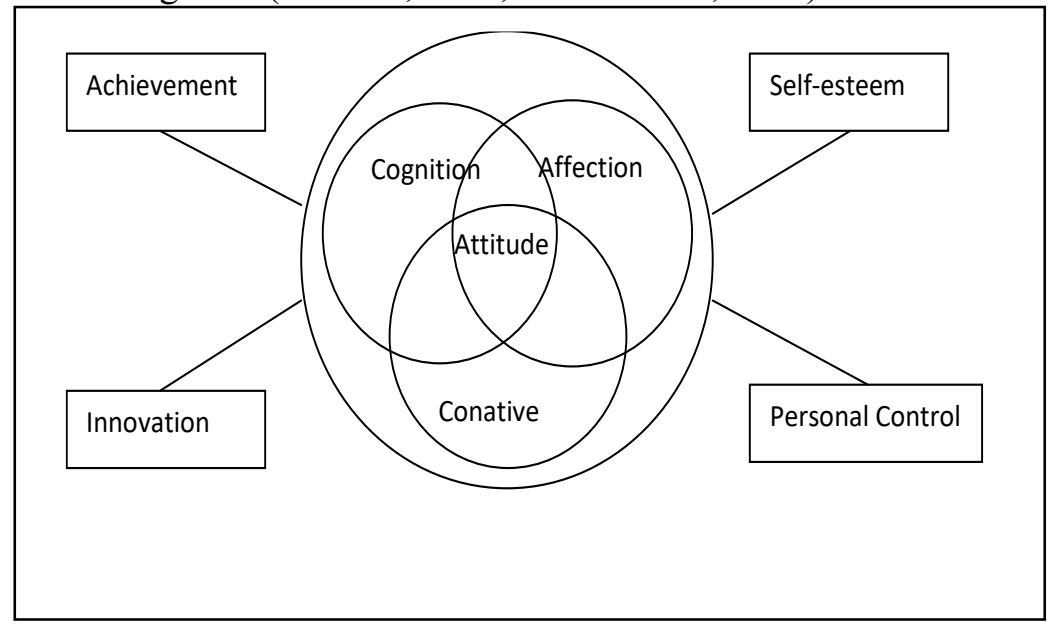

Hình 1: Mô hình thái độ định hướng khởi nghiệp

Như vậy, trong mô hình EAO của mình, tác giả Robinson đã xây dựng 12 khái niệm đo lường, gồm: (1)SE_AFF (cảm xúc tự tin), (2) SE_BEH (Hành vi tự tin), (3)SE_COG (nhận thức Sự tự tin), (4) PC_AFF (Cảm xúc kiểm soát cá nhân), (5) PC_BEH (Hành vi kiểm soát cá nhân), (6) PC_COG (Nhận thức khả năng kiểm soát cá nhân), (7)ACH_AFF (Cảm xúc về thành tựu), (8) ACH_BEH (Hành vi định hướng thành tựu), (9) ACH_COG (nhận thức về thành tựu), (10) INN_AFF (Cảm xúc sáng tạo), (11) INN_BEH (Hành vi sáng tạo), (12) INN_COG (Nhận thức về sáng tạo).

Thang đo $\mathrm{EAO}$ đã được sử dụng rộng rãi trong các nghiên cứu để đo lường các quan điểm về tinh thần kinh doanh (Stimpson, Huefner, Narayanan, \& Shanthakumar, 1993; Boshoff \& Scholtz, 1995; Wyk \& Boshoff, 2004; Harris \& Gibson, 2008; Sharif \& Saud, 2009). Tuy nhiên, có rất ít các nghiên cứu kiểm tra giá trị và độ tin cậy của mô hình $\mathrm{EAO}$ trước để kiểm chứng mối quan hệ của nó với các cấu trúc khác. Kêt quả này cho thấy sự cần thiết phải kiểm tra lại mô hình EAO trong các bối cảnh xã hội và văn hóa khác nhau.

Robinson cho rằng "chủ đề này là một lĩnh vực hứa hẹn tiềm năng cho các nhà nghiên cứu trong tương lai” (Robinson, et al., 1991). Cho đến nay, không có bất kỳ nghiên cứu thực nghiệm nào được tiến hành ở Việt Nam. Không chắc rằng liệu mô hình $\mathrm{EAO}$ có thể được ứng dụng ở Việt Nam hay không. Trong nghiên cứu này, mục đích của tác giả là để xem xét độ tin cậy và giá trị của thang đo thái độ định hướng kinh doanh trong bối cảnh tại Việt Nam.

\section{PHƯƠNG PHÁP}

Nghiên cứu này sử dụng phương pháp lấy mẫu thuận tiện để thu thập dữ liệu. Bảng câu hỏi có nguồn gốc bằng tiếng Anh và được dịch sang tiếng Việt. Sau đó, tác giả đã đề nghị người không cùng lĩnh vực dịch ngược lại sang Tiếng Anh và tiến hành so sánh để đảm bảo tính hợp lệ của các thang đo. Các câu hỏi đã được tiến hành thăm dò bằng 2 cách: một là gọi điện thoại giải thích và gởi link Google drives khảo sát 
qua email, hai là phỏng vấn trực tiếp. Nghiên cứu sẽ được tiến hành theo hai bước: một là nghiên cứu sơ bộ gồm nghiên cứu định tính và nghiên cứu định lượng; sau đó đưa vào nghiên cứu chính thức.

\subsection{Nghiên cứu so bộ}

Qui trình nghiên cứu sơ bộ được tiến hành qua 2 bước: nghiên cứu định tính và nghiên cứu định lượng. Trong phần nghiên cứu định tính, bảng câu hỏi sau khi được chuyển thể từ tiếng Anh sang tiểng Việt như đã trình bày ở trên sẽ được tiến hành đưa vào thảo luận nhóm. Phương pháp chọn mẫu trong bước nghiên cứu này là lấy mẫu thuận tiện, kỹ thuật dùng trong nghiên cứu định tính là phỏng vấn. Đối tượng tham gia phỏng vấn trong giai đoạn này được tác giả chọn là 20 cá nhân sống trên địa bàn thành phố Hồ Chí Minh. Trong phần nghiên cứu định lượng tác giả sử dụng phương pháp khảo sát trực tiếp với số phiếu phát ra: 60 , số phiếu hợp lệ thu về: 44. Đối tượng được khảo sát là người Việt Nam trên 17 tuổi và sống tại ba thành phố lớn Việt Nam là Hà Nội, Đà Nẵng và Hồ Chí Minh. Hệ số Cronbach alpha sẽ được ứng dụng để đánh giá độ tin cậy của các thang đo. Trong phân tích nhân tố, nhiều nhà nghiên cứu đồng ý rằng khi Cronbach alpha từ 0,8 trở lên gần đến 1 thì thang đo là tốt (Nunnally \& Bernstein, 1994). Từ 0,7 dến gần 0,8 là sử dụng được (Peterson, 1994). Có nhà nghiên cứu đề nghị rằng Cronbach alpha từ 0,6 trở lên là có thể sử dụng được trong trường hợp khái niệm đang nghiên cứu là mới đối với người trả lời trong bối cảnh nghiên cứu (Slater, 1995).

Trong đánh giá độ tin cậy thang đo, cần ghi nhận rằng Cronbach alpha đo lường độ tin cậy của cả thang đo (Nguyễn Đình Thọ, 2011) chứ không tính độ tin cậy cho từng biến quan sát. Hơn thế, các biến trong cùng một thang đo dùng để đo lường cùng một khái niệm nghiên cứu nên chúng phải có tương quan chặt chẽ với nhau. Vì vậy, khi kiểm tra từng biến đo lường nguời ta sử dụng hệ số tương quan biến tổng (Nguyễn Đình Thọ, 2011). Hệ số tin cậy Cronbach's Alpha chỉ cho biết các đo lường có liên kết với nhau hay không; nhưng không cho biết biến quan sát nào cần bỏ đi và biến quan sát nào cần giữ lại. Khi đó, việc tính toán hệ số tương quan giữa biến-tổng sẽ giúp loại ra những biến quan sát nào không đóng góp nhiều cho sự mồ tả của khái niệm cần đo (Hoàng Trọng \& Chu Nguyễn Mộng Ngọc, 2005. Có nhiều quan điểm loại biến khác nhau, song tác giả chọn tiêu chí các biến quan sát có hệ số tương quan biến tổng (hiệu chỉnh) $>=0.4$ và Cronbach alpha $>=0.6$, trường hợp biến có tương quan biến tổng $(0.3<\mathrm{X}<0.4)$ nhưng là biến có ý nghĩa sẽ được kiến nghị giữ lại để kiểm tra trong nghiên cứu chính thức với mẫu lớn hơn.

\subsection{Nghiên cứu chính thức}

Chương trình nghiên cứu chính thức bằng phương pháp nghiên cứu định lượng được thực hiện bằng cách khảo sát trực tiếp với số phiếu phát ra: 400, số phiếu hợp lệ thu về: 337 . Đối tượng được khảo sát là người Việt Nam trên 17 tuổi và sống tại ba thành phố lớn Việt Nam là Hà Nội, Đà Nẵng và Hồ Chí Minh.

Hệ số Cronbach alpha sẽ được ứng dụng để đánh giá độ tin cậy của các thang đo. Sau đó, phương pháp phân tích nhân tố khám phá EFA sẽ được dùng để đo lường giá trị thang đo.

\section{KÊT QUẢ VÀ THẢO LUẬN}

\subsection{Kết quả nghiên cứu so bộ}

Qua triển khai chương trình nghiên cứu sơ bộ, loại bỏ các biến trùng lắp hoặc không phù hợp trong bối cảnh nghiên cứu tại Việt Nam các thang đo đã được điều chỉnh có độ tin cậy cao và thích hợp hơn trước khi đưa vào nghiên cứu chính thức. Việc có nhiều biến bị loại xuất phát từ hai lý do chính: một là, các câu hỏi nguyên bản từ tiếng Anh khi được chuyển ngữ sẽ có độ "nhiễu" nhất định, có thể gây ra hiện tượng trùng lắp, khó hiểu hoặc không phù hợp bối cảnh; hai là, bản thân thang đo EAO gồm 75 câu hỏi là khá dài, nhiều mục được đo lường bởi 9 câu hỏi khác nhau khiến việc trả lời không đạt giá trị chính xác cao. Điều này cho thấy thang đo EAO cần được hiệu chỉnh cũng như kiểm chứng nhiều hơn tại các nền văn hóa khác nhau. Các thang đo sau khi hiệu chỉnh sơ bộ được trình bày cụ thể dưới đây sẽ được đưa vào làm bảng câu hỏi trong nghiên cứu chính thức (Phụ lục 1). 


\subsection{Kết quả nghiên cứu chính thức}

\subsection{1 Đánh giá độ tin cậy của thang đo bằng Cronbach alpha}

Bảng 1: Đánh giá độ tin cậy thang đo SE

\begin{tabular}{|l|r|r|r|r|r|}
\hline SE_AFF & \multicolumn{2}{|l|}{$\begin{array}{l}\text { Trung bình thang đo } \\
\text { nếu loại biến }\end{array}$} & $\begin{array}{l}\text { ne̛ơng sai thang đo } \\
\text { nếu loại biến } \\
\text { biến tổng }\end{array}$ & $\begin{array}{l}\text { quan Cronbach } \alpha \text { nếu loại } \\
\text { biến }\end{array}$ \\
\hline SE_AFF1 & 18.61 & 26.025 & .415 & .735 \\
\hline SE_AFF2 & 18.18 & 23.546 & .561 & .682 \\
\hline SE_AFF3 & 18.14 & 22.535 & .594 & .668 \\
\hline SE_AFF4 & 18.82 & 25.562 & .435 & .728 \\
\hline SE_AFF5 & 17.91 & 23.653 & .547 & .688 \\
\hline
\end{tabular}

Cronbach's Alpha $=.746(\mathrm{~N}=5)(>.0 .6)$ và tương quan biến tổng của tất cả các biến trong thang đo này biến thiên từ 0.415 đến 0.594 , đều $>0.4$. Như vậy thang đo đáp ứng độ tin cậy cần thiết.

\begin{tabular}{|l|r|r|r|r|}
\hline Thang do SE_BEH & & 12.745 & -.090 & .499 \\
\hline SE_BEH1 & 13.81 & 11.452 & -.001 & .436 \\
\hline SE_BEH2 & 12.76 & 6.693 & .435 & $-.154^{\mathrm{a}}$ \\
\hline SE_BEH3 & 12.72 & 7.409 & .384 & $-.048^{\mathrm{a}}$ \\
\hline SE_BEH4 & &
\end{tabular}

Cronbach's Alpha $=.319(\mathrm{~N}=4)$.

Kết quả cho thấy thang đo hành vi tự trọng được đo lường bởi 4 biến quan sát. Kết quả phân tích ban đầu cho thấy hệ số Cronbach’s alpha = .319 $(<<0.6)$ vẫn không đảm bảo độ tin cậy. Quan sát cột “Cronbach's alpha nếu loại biến” thấy rằng trị số $\alpha$ của tất các biến quan sát đều thấp $(<<0.6)$. Do đó, tác giả quyết định loại biến "Hành vi tự trọng” do không phù hợp tại bối cảnh nghiên cứu ở Việt Nam.

\section{Thang đo SE_COG}

Lần $1($ Cronbach's Alpha $=.691(\mathrm{~N}=4)$

\begin{tabular}{|l|r|r|r|r|}
\hline SE_COG1 & 15.43 & 10.495 & .577 & .555 \\
\hline SE_COG2 & 15.57 & 11.114 & .607 & .545 \\
\hline SE_COG3 & 16.46 & 13.237 & .267 & .757 \\
\hline SE_COG4 & 15.27 & 11.890 & .486 & .619 \\
\hline
\end{tabular}

Lần $2($ Cronbach's Alpha $=.757(\mathrm{~N}=3)$

\begin{tabular}{|l|r|r|r|r|}
\hline SE_COG1 & 10.98 & 5.904 & .638 & .614 \\
\hline SE_COG2 & 11.12 & 6.863 & .591 & .671 \\
\hline SE_COG4 & 10.82 & 7.028 & .536 & .730 \\
\hline
\end{tabular}

Sau khi loại biến SE_COG3, tính toán lại độ tin cậy của thang đo, có Crobach’s alpha $=.746(>.7)$ và tương quan biến tổng của tất cả các biến trong thang đo này biến thiên từ 0.536 dến 0.638 , đều $>0.4$. Nhu vậy thang đo đáp ứng độ tin cậy cần thiết. 
Bảng 2: Đánh giá độ tin cậy thang đo PC

\begin{tabular}{|c|c|c|c|c|}
\hline PC_AFF & $\begin{array}{l}\text { Trung bình thang đo } \\
\text { nếu loại biến }\end{array}$ & $\begin{array}{c}\text { Phương sai thang đo } \\
\text { nếu loại biến }\end{array}$ & $\begin{array}{l}\text { Tương quan biến } \\
\text { tổng }\end{array}$ & $\begin{array}{c}\text { Cronbach } \alpha \text { nếu loại } \\
\text { biến }\end{array}$ \\
\hline \multicolumn{5}{|c|}{ Lần $1($ Cronbach's Alpha $=.604(\mathrm{~N}=3)$} \\
\hline PC_AFF1 & 11.57 & 5.562 & .229 & .803 \\
\hline PC_AFF2 & 10.63 & 5.109 & .502 & .379 \\
\hline PC_AFF3 & 10.37 & 5.031 & .565 & .300 \\
\hline \multicolumn{5}{|c|}{ Lần $2($ Cronbach's Alpha $=.810(\mathrm{~N}=2)$} \\
\hline PC_AFF2 & 5.90 & 1.595 & .680 & $\cdot{ }^{\mathrm{a}}$ \\
\hline PC_AFF3 & 5.66 & 1.677 & .680 & $a^{a}$ \\
\hline
\end{tabular}

Sau khi loại PC_AFF1, độ tin cậy của thang đo có Crobach's alpha $=.810(>0.7)$ và tương quan biến tổng của tất cả các biến trong thang đo này là $.680>0.4$. Như vậy thang đo đáp ứng độ tin cậy cần thiết.

\section{Thang đo PC_BEH}

PC BEH

PC_BEH4

PC_BEH5

PC_BEH6

\begin{tabular}{r|r|r|r|}
15.16 & 10.591 & .431 & .689 \\
\hline 15.82 & 11.143 & .391 & .710 \\
\hline 15.65 & 9.426 & .573 & .601 \\
\hline 15.37 & 9.323 & .607 & .580 \\
\hline
\end{tabular}

Cronbach's Alpha = .712 $(\mathrm{N}=4)$, hệ số tương quan biến tổng của các biến quan sát đều lớn hơn 0.4. Nhu vậy thang đo đáp ứng độ tin cậy cần thiết.

\section{Thang đo PC_COG}

\begin{tabular}{|l|r|r|r|r|}
\hline PC_COG2 & 5.24 & 2.296 & .486 &. a \\
\hline PC_COG3 & 5.09 & 2.216 & .486 &. a \\
\hline
\end{tabular}

Kết quả phân tích độ tin cậy cho thấy hệ số Cronbach's alpha $=0.654(>0.6)$. Trường hợp này, khái niệm "nhận thức khả năng kiểm soát cá nhân" được các chuyên gia đánh giá là một khái niệm mới trong nghiên cứu khởi nghiệp, vì vậy giá trị $\alpha>0.6$ được cho là chấp nhận được, tương quan biến tổng đều lớn hơn 0.4. Như vậy thang đo có độ tin cậy chấp nhận được.

Bảng 3: đánh giá độ tin cậy thang đo $\mathrm{ACH}$

\begin{tabular}{|c|c|c|c|c|}
\hline ACH_AFF & $\begin{array}{l}\text { Trung bình thang đo } \\
\text { nếu loại biến }\end{array}$ & $\begin{array}{c}\text { Phương sai thang đo } \\
\text { nếu loại biến }\end{array}$ & $\begin{array}{l}\text { Tương quan biến } \\
\text { tổng }\end{array}$ & $\begin{array}{l}\text { Cronbach } \alpha \text { nếu loại } \\
\text { biến }\end{array}$ \\
\hline ACH_AFF3 & 17.16 & 11.147 & .654 & .842 \\
\hline ACH_AFF5 & 17.14 & 11.751 & .728 & .811 \\
\hline ACH_AFF6 & 17.19 & 11.148 & .730 & .808 \\
\hline ACH_AFF7 & 17.04 & 11.501 & .708 & .817 \\
\hline \multicolumn{5}{|c|}{$\begin{array}{l}\text { Cronbach's Alpha = .858 (N=4), hệ số tương quan biến tổng của tất cả các biến quan sát đều lớn (>>0.4). } \\
\text { Như vậy thang đo có giá trị tin cậy cao hơn. }\end{array}$} \\
\hline \multicolumn{5}{|c|}{ Thang do ACH_BEH } \\
\hline ACH_BEH1 & 25.56 & 22.384 & .442 & .808 \\
\hline
\end{tabular}




\begin{tabular}{|l|r|r|r|r|}
\hline ACH_BEH2 & 25.36 & 21.845 & .583 & .774 \\
\hline ACH_BEH3 & 25.40 & 21.569 & .575 & .776 \\
\hline ACH_BEH4 & 25.03 & 21.868 & .612 & .768 \\
\hline ACH_BEH5 & 24.98 & 21.401 & .650 & .759 \\
\hline ACH_BEH6 & 25.13 & 22.031 & .558 & .779 \\
\hline COn & & &
\end{tabular}

Cronbach's Alpha $=.808$, thang đo $\mathrm{ACH} \_\mathrm{BEH}$ được đo bằng 6 biến quan sát, tất cả các biến đều có tương quan biến tổng $>0.4$ nên thang đo này đạt độ tin cậy cao.

\begin{tabular}{|l|r|r|r|r|}
\hline Thang do ACH_COG & \\
\hline ACH_COG3 & 27.36 & 27.721 & .718 & .819 \\
\hline ACH_COG4 & 27.37 & 27.788 & .726 & .817 \\
\hline ACH_COG5 & 27.53 & 28.661 & .647 & .832 \\
\hline ACH_COG6 & 27.56 & 28.932 & .689 & .825 \\
\hline ACH_COG7 & 27.52 & 30.119 & .587 & .843 \\
\hline ACH_COG8 & 27.76 & 30.991 & .508 & .857 \\
\hline Croch & & &
\end{tabular}

Cronbach's Alpha $=.857(\mathrm{~N}=6)$, tất cả 6 biến quan sát đều có tương quan biến tổng $>0.4$ nên thang đo ACH_COG đạt độ tin cậy cao.

Bảng 4: Đánh giá độ tin cậy thang đo INN

\begin{tabular}{|l|r|r|r|r|}
\hline Thang đo INN_AFF & $\begin{array}{l}\text { Trung bình thang đo } \\
\text { nếu loại biến }\end{array}$ & $\begin{array}{l}\text { Phương sai thang đo } \\
\text { nếu loại biến }\end{array}$ & $\begin{array}{l}\text { Tương quan biến } \\
\text { tổng }\end{array}$ & \multicolumn{2}{l|}{$\begin{array}{l}\text { Cronbach Alpha nếu } \\
\text { loại biến }\end{array}$} \\
\hline INN_AFF3 & 16.32 & 10.092 & .453 & .746 \\
\hline INN_AFF7 & 15.91 & 10.110 & .510 & .715 \\
\hline INN_AFF8 & 16.13 & 9.009 & .658 & .634 \\
\hline INN_AFF9 & 16.32 & 8.842 & .583 & .675 \\
\hline
\end{tabular}

Cronbach's Alpha $=.752(\mathrm{~N}=4)$, tất cả 4 biến quan sát đều có tương quan biến tổng $>0.4$ nên thang đo INN_AFF đạt độ tin cậy cần thiết.

\section{Thang đo INN_BEH ( Hành vi sáng tạo)}

\begin{tabular}{|l|r|r|r|r|}
\hline INN_BEH1 & 23.40 & 22.431 & .439 & .703 \\
\hline INN_BEH2 & 23.74 & 20.994 & .502 & .684 \\
\hline INN_BEH3 & 23.75 & 22.463 & .440 & .702 \\
\hline INN_BEH4 & 23.34 & 21.672 & .546 & .671 \\
\hline INN_BEH5 & 23.10 & 23.671 & .431 & .704 \\
\hline INN_BEH6 & 22.86 & 22.968 & .444 & .700 \\
\hline
\end{tabular}

Trong phần nghiên cứu sơ bộ, thang đo INN_BEH mặc dù đạt độ tin cậy cần thiết song cần đánh giá lại biến quan sát INN_BEH6 do có hệ số lớn hơn Cronbach alpha tổng, nhưng hệ số tương quan biến tổng tương đối lớn. Qua nghiên cứu chính thức cho thấy thang đo hành vi sáng tạo INN_BEH gồm 6 biến quan sát có hệ số Cronbach's Alpha = 0.732, với tương quan biến tổng đều lớn hơn 0.4 nên đạt độ tin cậy. 


\begin{tabular}{|l|r|r|r|r|}
\hline Thang đo INN_COG (Nhận thức khả năng sáng tạo) \\
\hline INN_COG1 & 30.48 & 31.054 & .539 & .769 \\
\hline INN_COG2 & 30.79 & 32.424 & .487 & .778 \\
\hline INN_COG3 & 30.18 & 31.331 & .590 & .760 \\
\hline INN_COG5 & 30.09 & 31.854 & .542 & .768 \\
\hline INN_COG6 & 30.57 & 31.401 & .494 & .778 \\
\hline INN_COG7 & 30.58 & 32.381 & .518 & .773 \\
\hline INN_COG9 & 30.23 & 31.756 & .525 & .771 \\
\hline INOn & & & \\
\hline
\end{tabular}

Cronbach's Alpha $=.797(\mathrm{~N}=7)$. INN_COG được đo lường bởi 7 biến quan sát có tương quan biến tổng $>0.4$ và do đó đạt độ tin cậy cần thiết.

Sau khi tiến hành kiểm định độ tin cậy thang đo, kết quả cho thấy hầu hết hệ số Cronbach alpha của các thang đo đều đạt độ tin cậy. Riêng thang đo SE_BEH không thể hiện giá trị tin cậy trong bối cảnh nghiên cứu tại Việt nam (Cronbach's Alpha $=0.319)$. Ngoài ra, việc loại biến quan sát PC_AFF1 và SE_COG3 giúp làm tăng giá trị thang đo.

\subsubsection{Kiểm định giá trị hội tụ và phân biệt của thang đo}

Phương pháp phân tích nhân tố khám phá (EFA) sẽ được dùng để đánh giá giá trị thang đo (Bảng 5).

Bảng 5: Giá trị KMO và Barlett biến độc lập

\begin{tabular}{|l|l|l|}
\hline KMO and Bartlett's Test & .850 \\
\hline Kaiser-Meyer-Olkin Measure of Sampling Adequacy. & $4.412 \mathrm{E} 3$ \\
\hline \multirow{3}{*}{ Bartlett's Test of Sphericity } & Approx. Chi-Square & 630 \\
\cline { 2 - 3 } & Df & .000 \\
\cline { 2 - 3 } & Sig. & \\
\hline
\end{tabular}

Kết quả phân tích nhân tố cho thấy chỉ số KMO là 0.85 và phép kiểm định Bartlett có giá trị sig = 0.000 . Như vậy kết quả phân tích nhân tố là thích hợp.

Bảng 6: Bảng tổng phương sai trích

\begin{tabular}{|c|c|c|c|c|c|c|c|c|c|}
\hline \multirow[b]{2}{*}{$\begin{array}{c}\text { Thành } \\
\text { phần }\end{array}$} & \multicolumn{3}{|c|}{ Eigenvalues khởi tạo } & \multicolumn{3}{|c|}{$\begin{array}{l}\text { Extraction Sums of Squared } \\
\text { Loadings }\end{array}$} & \multicolumn{3}{|c|}{$\begin{array}{c}\text { Rotation Sums of Squared } \\
\text { Loadings }\end{array}$} \\
\hline & Tổng & $\begin{array}{c}\% \text { phương } \\
\text { sai }\end{array}$ & $\%$ tích lũy & Tổng & $\begin{array}{c}\text { \% phương } \\
\text { sai }\end{array}$ & $\%$ tích lũy & Tổng & $\begin{array}{c}\% \text { phương } \\
\text { sai }\end{array}$ & $\%$ tích lũy \\
\hline 1 & 8.434 & 23.429 & 23.429 & 8.434 & 23.429 & \begin{tabular}{|r|}
23.429 \\
\end{tabular} & 3.751 & \begin{tabular}{|l|}
10.419 \\
\end{tabular} & 10.419 \\
\hline 2 & 2.769 & 7.691 & 31.120 & 2.769 & 7.691 & 31.120 & 2.797 & 7.768 & 18.187 \\
\hline 3 & 2.251 & 6.253 & 37.373 & 2.251 & 6.253 & 37.373 & 2.561 & 7.113 & 25.300 \\
\hline 4 & 1.624 & 4.510 & 41.883 & 1.624 & 4.510 & 41.883 & 2.449 & 6.801 & 32.101 \\
\hline 5 & 1.511 & 4.199 & 46.081 & 1.511 & 4.199 & 46.081 & 2.206 & 6.129 & 38.230 \\
\hline 6 & 1.391 & 3.864 & 49.945 & 1.391 & 3.864 & 49.945 & 2.154 & 5.985 & 44.214 \\
\hline 7 & 1.293 & 3.592 & 53.537 & 1.293 & 3.592 & 53.537 & 1.966 & 5.462 & 49.677 \\
\hline 8 & 1.207 & 3.352 & 56.890 & 1.207 & 3.352 & 56.890 & 1.962 & 5.450 & 55.127 \\
\hline 9 & 1.095 & 3.042 & 59.932 & 1.095 & 3.042 & 59.932 & 1.730 & 4.805 & 59.932 \\
\hline
\end{tabular}


Tại nhân tố thứ 9 , giá trị Eigenvalue là $1.095(>1)$, nhân tố thứ 10 có giá trị Eigenvalue là $0.978(<1)$. Như vậy, điểm dừng trích nhân tố tại nhân tố thứ 9 . Tổng phương sai trích được là $59.932 \%(=60 \%)$, như vậy, mô hình EFA là phù hợp.

Bảng 7: Ma trận xoay nhân tố

\begin{tabular}{|c|c|c|c|c|c|c|c|c|c|}
\hline & & & & & Nhât & & & & \\
\hline & 1 & 2 & 3 & 4 & 5 & 6 & 7 & 8 & 9 \\
\hline ACH_AFF3 & .735 & & & & & & & & \\
\hline ACH_AFF5 & .708 & & & & & & & & \\
\hline ACH_AFF6 & .660 & & & & & & & & \\
\hline PC_AFF3 & .607 & & & & & & & & \\
\hline PC_AFF2 & .501 & & & & & & & & \\
\hline PC_COG3 & & .685 & & & & & & & \\
\hline ACH_COG7 & & .598 & & & & & & & \\
\hline ACH_COG6 & & .595 & & & & & & & \\
\hline PC_COG2 & & .564 & & & & & & & \\
\hline ACH_COG8 & & .556 & & & & & & & \\
\hline ACH_COG5 & & .547 & & & & & & & \\
\hline SE_AFF3 & & & .769 & & & & & & \\
\hline SE_AFF2 & & & .728 & & & & & & \\
\hline SE_AFF5 & & & .720 & & & & & & \\
\hline SE_AFF4 & & & .650 & & & & & & \\
\hline SE_AFF1 & & & .626 & & & & & & \\
\hline INN_COG2 & & & & .741 & & & & & \\
\hline INN_COG1 & & & & .720 & & & & & \\
\hline INN_COG3 & & & & .610 & & & & & \\
\hline INN_COG7 & & & & .578 & & & & & \\
\hline INN_COG6 & & & & .577 & & & & & \\
\hline INN_AFF3 & & & & & .689 & & & & \\
\hline INN_AFF9 & & & & & .660 & & & & \\
\hline INN_AFF8 & & & & & .591 & & & & \\
\hline INN_BEH2 & & & & & & .763 & & & \\
\hline INN_BEH3 & & & & & & .743 & & & \\
\hline INN_BEH4 & & & & & & .697 & & & \\
\hline INN_BEH1 & & & & & & .581 & & & \\
\hline ACH_BEH1 & & & & & & & .765 & & \\
\hline ACH_BEH2 & & & & & & & .727 & & \\
\hline ACH_BEH3 & & & & & & & .581 & & \\
\hline PC_BEH5 & & & & & & & & .819 & \\
\hline PC_BEH6 & & & & & & & & .640 & \\
\hline PC_BEH4 & & & & & & & & .638 & \\
\hline SE_COG2 & & & & & & & & & .794 \\
\hline SE_COG1 & & & & & & & & & .738 \\
\hline
\end{tabular}


Kết quả phân tích nhân tố cho thấy có 9 nhân tố được trích ra từ 11 yếu tố ban đầu được đưa vào và phương sai trích là $59.93 \%$ (giải thích được $59.93 \%$ sự biến thiên của dữ liệu).

Dựa vào bảng kết quả ma trận xoay nhân tố ta thấy nhân tố $\mathrm{ACH}$ _AFF và PC_AFF kết hợp lại với nhau, nhân tố PC_COG và ACH_COG kết hợp lại với nhau do cùng tải trên 1 nhân tố. Ngoài ra, do cỡ mẫu là $337(<350)$ nên trọng số nhân tố được chọn là 0.5 , kết quả cho thấy trọng số nhân tố đều thỏa (> 0.5). Thang đo EAO gồm có 5 thành phần gồm: (1) $\mathrm{SE}$, (2) $\mathrm{PC}$, (3) $\mathrm{ACH}$, (4) INN và (5) ACHPC.

Như vậy sau khi phân tích nhân tố, thang đo EAO có "giá trị hội tụ" (các items hội tụ về cùng 1 nhân tố) và đảm bảo "Giá trị phân biệt" (thuộc về nhân tố này và phải phân biệt với nhân tố khác).

\section{KẾT LUẬN}

Sau khi được tiến hành đánh giá và điều chỉnh tại Việt Nam, kết quả cho thấy thang đo EAO gồm có 11 thang đo thành phần thay vì 12 như nguyên bản, với việc loại thành phần $\mathrm{SE}$ BEH, thang đo EAO gồm 49 câu hỏi, đảm bảo tính hội tụ và phân biệt. Kết quả của quá trình đánh giá này đã sàng lọc những câu hỏi không phù hợp trong các thang đo trong bối cảnh nghiên cứu tại Việt Nam. Nghiên cứu này là một trong những nỗ lực đầu tiên để kiểm tra độ tin cậy và tính xác thực của mô hình $\mathrm{EAO}$ ở Việt Nam. Thang đo đề xuất gồm 49 câu hỏi đã được điều chỉnh có thể được sử dụng như là một phát hiện để đánh giá thái độ kinh doanh của các doanh nhân tiềm năng cũng như các doanh nhân hiện tại, đặc biệt phù hợp hơn trong bối cảnh của Việt Nam. Kết quả này có khác biệt so với đề xuất của tác giả Robinson. Đồng thời cũng khác so với nghiên cứu của Wyk và Boshoff's(2004) khi tiến hành tại Nam Phi với kết quả chỉ có 3 thành phần thay vì 4 thành phần, trong đó $\mathrm{PC}$ và $\mathrm{ACH}$ nhập lại với nhau. $\mathrm{Ngoài} \mathrm{ra,} \mathrm{thang} \mathrm{đo} \mathrm{EAO}$ chỉ gồm 62 câu hỏi sau khi loại đi 13 câu do không thỏa mãn độ tin cậy dù các tác giả không chỉ ra cụ thể lý do.

Bởi vì nghiên cứu của chúng tôi sử dụng cách tiếp cận lấy mẫu thuận tiện nên các nghiên cứu trong tương lai nên xem lại độ tin cậy của thang đo $\mathrm{EAO}$ giữa các nhóm đối tượng cụ thể, chẳng hạn: sinh viên đại học, học viên thạc sĩ, người đi làm, các doanh nhân... Cần phải sử dụng thang EAO điều chỉnh để tìm hiểu liệu thái độ đối với tinh thần kinh doanh có thay đổi theo thời gian hay không. Ngoài ra, do kết quả có sự khác biệt với nguyên bản của Robinson (1991) và các nghiên cứu khác cho thấy thang đo cần phải được tiếp tục kiểm chứng tại các bối cảnh nghiên cứu khác nhau. Hơn nữa, cần phải xem xét giá trị thực tiển của thang đo này trong việc dự báo việc ra quyết định kinh doanh của các cá nhân.

\section{TÀI LIỆU THAM KHẢO}

1. Abelson, R. P. (1982). Three models of attitude-behavior consistency. In M. P. Zanna, E. T. Higgins, \& C. P. Herman (Eds.), Consistency in social behavior: The Ontario symposium, vol. 2, pp. 131146. Hillsdale, NJ: Erlbaum

2. Ajzen, I. (1991). The theory of planned behavior. Organizational Behavior and Human Decision Processes, 50(2), 179-211

3. Boshoff, A.B., \& Scholtz, C. P. T. (1995) Measuring attitudes as a way of differentiating entrepreneurs. South African Journal of Economics and Management Science, 16, 1-11.

4. Chaiken, S., \& Stangor, C. (1987). Attitudes and attitude change. Annual Review oj Psychology.

5. Fayolle, A. and Klandt, H. (2006a), International Entrepreneurship Education. Issues and Newness, Edward Elgar Publishing, Aldershot.

6. Fayolle, Gailly, \& Lassas-Clerc, 2006b. Accessing the impact of entrepreneurship education programs: a new methodology. Journal of European Industrial Training, 30(9), 701-720.

7. Harris, M.L., \& Gibson, S.G. (2008) Examining the entrepreneurial attitudes of U.S. business students. Education + Training, 50, 568-561.

8. Huefner, J., Hunt, H. K., and Robinson, P. B., (1996). Comparison of four scales predicting entrepreneurship. Academy of Entrepreneurship Journal, Fall, Vol. 1(2), 56Đ80.

9. Kermit, W. (2008). Entrepreneurial intention research: Implications for entrepreneurship education. Journal of Entrepreneurship Education, Annual 2008

10. Kollmann, T., \& Kuckertz, A. (2006). Venture archetypes and the entrepreneurial event: cross-cultural empirical evidence. Journal of Enterprising Culture, 14(1): 27-48.

11. Rosenberg, M. J., \& Hovland, C. I. (1960). Cognitive, affective, and behavioral components of attitudes. In M. J. Rosenberg, C. I. Hovland, W. J. McGuire, R. P. Abelson, \& J. W. Brehm 
(Eds.), Attitude organization and change: An analysis of consistency among attitude components. New Haven, CT: Yale University.

12. Stimpson, V.S., Huefner, J. C., Narayanan, S., \& Shanthakumar, D. (1993): Attitudinal characteristics of male and female entrepreneurs of the United States and India. Psychological Studies, 38, 64-68.

13. Shapero, A. \& Sokol, L. (1982). Social dimensions of entrepreneurship. In C.A. Kent, D.L. Sexton, \& K.H. Vesper (Eds.), the encyclopedia of entrepreneurship (pp. 72-90). Englewood Cliffs, NJ: Prentice-Hall

14. Shariff, M.N., \& Saud, M.B. (2009) An attitude approach to the prediction of entrepreneurship on students at institution of higher learning in Malaysia. International Journal of Business and Management, 4, 129135.

15. Robinson, P. B., Stimpson, D.V, Huefner, J. C., \& Hunt, H.K. (1991). An attitude approach to the prediction of entrepreneurship. Entrepreneurship Theory and Practice, 15, 13-31.

16. Wyk, R.V., \& Boshoff, A. B. (2004) Entrepreneurial attitudes: a distinction between two professional groups. South African Journal of Business Management, 35, 33-38.

Ngày nhận bài: 25/12/2017

Ngày chấp nhận đăng: 10/04/2018 


\section{Phụ lục 1: Bảng câu hỏi khảo sát}

\begin{tabular}{|c|c|}
\hline \multicolumn{2}{|c|}{ Thang đo cảm xúc tự trọng (SE-AFF) } \\
\hline SE-AFF1 & Tôi cảm thấy như một thất bại khi kế hoạch kinh doanh không diễn ra như tôi mong muốn. \\
\hline SE-AFF2 & Tôi cảm thấy không thoải mái khi làm đề án kinh doanh. \\
\hline SE-AFF3 & Tôi cảm thấy tự ti khi gặp những doanh nhân thành đạt. \\
\hline SE-AFF4 & Tôi cảm thấy không thoải mái khi không rõ các đồng nghiệp nghĩ gì về tôi. \\
\hline SE-AFF5 & Tôi cảm thấy thua kém hầu hết những người tôi làm việc cùng. \\
\hline \multicolumn{2}{|c|}{ Thang đo hành vi tự trọng (SE-BEH) } \\
\hline SE-BEH1 & $\begin{array}{l}\text { Tôi thường xuyên thực hiện rất tốt phần công việc của mình trong bất kì dự án kinh doanh } \\
\text { nào tôi tham gia. }\end{array}$ \\
\hline SE-BEH2 & $\begin{array}{l}\text { Tôi hay dành nhiều thời gian tìm kiếm người nào đó có thể nói cho tôi cách giải quyết mọi } \\
\text { vấn đề trong việc kinh doanh của tôi. }\end{array}$ \\
\hline SE-BEH3 & Tôi thường “diễn” để gây ấn tượng với đồng nghiệp. \\
\hline SE-BEH4 & Tôi chưa bao giờ kiên nhẫn đủ lâu để giải quyết một công việc khó khăn trước khi từ bỏ. \\
\hline \multicolumn{2}{|c|}{ Thang đo nhận thức tự trọng (SE-COG) } \\
\hline SE-COG1 & $\begin{array}{l}\text { Tôi tin rằng những người thành công có khả năng xã giao tốt ở những buổi gặp gỡ kinh } \\
\text { doanh. }\end{array}$ \\
\hline SE-COG2 & $\begin{array}{l}\text { Tôi tin rằng để thành công trong kinh doanh thì điều quan trọng là phải hòa đồng với } \\
\text { những người làm việc cùng. }\end{array}$ \\
\hline SE_COG3 & $\begin{array}{l}\text { Tôi tin rằng quyền hạn có được trong kinh doanh chủ yếu là vì chuyên môn của tôi trong } \\
\text { lĩnh vực đó. }\end{array}$ \\
\hline SE-COG4 & Tôi tin rằng việc gây ấn tượng đầu tiên là rất quan trọng. \\
\hline \multicolumn{2}{|c|}{ Thang đo cảm xúc kiểm soát bản thân (PC-AFF) } \\
\hline PC-AFF1 & Tôi cảm thấy bực bội khi bị hạch sách ở nơi làm việc. \\
\hline PC-AFF2 & $\begin{array}{l}\text { Tôi cảm thấy thật tuyệt vời khi tôi hoàn toàn chịu trách nhiệm cho sự thành công của việc } \\
\text { kinh doanh của tôi. }\end{array}$ \\
\hline PC-AFF3 & Tôi phấn khích khi tạo ra cơ hội kinh doanh cho mình. \\
\hline \multicolumn{2}{|c|}{ Thang đo hành vi kiểm soát bản thân (PC-BEH) } \\
\hline PC-BEH1 & Tôi luôn nỗ lực để trở thành một trong nhữn \\
\hline PC-BEH4 & oạt động kinh doanh. \\
\hline PC-BEH5 & $\begin{array}{l}\text { Tôi tích cực tham gia vào các hoạt động cộng đồng để có thể tác động đến các sự kiện có } \\
\text { khả năng ảnh hưởng đến việc kinh doanh. }\end{array}$ \\
\hline PC-BEH6 & Khả năng làm việc với con người đã giúp tôi tạo ra rất nhiều cơ hội kinh doanh. \\
\hline \multicolumn{2}{|c|}{ Thang đo nhận thức kiểm soát bản thân (PC-COG) } \\
\hline PC-COG2 & $\begin{array}{l}\text { Tôi tin rằng trong giới kinh doanh, thành quả công việc của những người có năng lực tốt sẽ } \\
\text { luôn được công nhận. }\end{array}$ \\
\hline PC-COG3 & $\begin{array}{l}\text { Tôi tin rằng mọi tồ chức sẽ hoạt động hiệu quả hơn bằng cách tuyển dụng người có năng } \\
\text { lực tốt. }\end{array}$ \\
\hline \multicolumn{2}{|c|}{ Thang đo cảm xúc về thành công (ACH-AFF) } \\
\hline ACH-AFF3 & Tôi tự hào khi nhìn thấy những kết quả đạt được trong hoạt động kinh doanh của mình. \\
\hline ACH-AFF5 & Tôi thấy vui khi làm việc chăm chỉ để cải thiện việc kinh doanh của mình. \\
\hline ACH-AFF6 & Tôi thấy mãn nguyện khi theo đuổi các cơ hội kinh doanh của mình. \\
\hline ACH-AFF7 & Tôi luôn thấy vui khi làm cho các tổ chức mình tham gia hoạt động tốt hơn. \\
\hline \multicolumn{2}{|c|}{ Thang đo hành vi định hướng thành công (ACH-BEH) } \\
\hline ACH-BEH1 & $\begin{array}{l}\text { Tôi không bao giờ trì hoãn một vấn đề quan trọng tới một thời điểm thuận tiện hơn trong } \\
\text { tương lai. }\end{array}$ \\
\hline ACH-BEH2 & $\begin{array}{l}\text { Tôi dành một khoảng thời gian đáng kể để giúp tổ chức nơi tôi đang làm việc hoạt động } \\
\text { tốt hơn. }\end{array}$ \\
\hline $\mathrm{ACH}-\mathrm{BH}$ & 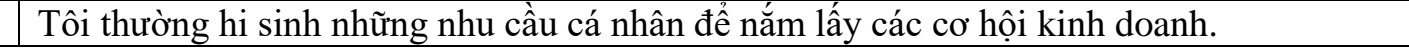 \\
\hline
\end{tabular}




\begin{tabular}{|c|c|}
\hline ACH-BEH4 & \\
\hline ACH-BEH5 & $\begin{array}{l}\text { Tôi nỗ lực triệt để để đạt được hiệu quả sử dụng các nguồn lực kinh doanh của mình cao } \\
\text { nhất. }\end{array}$ \\
\hline ACH-BEH6 & $\begin{array}{l}\text { Tôi sẽ dành một số lượng đáng kể thời gian để phân tích các nhu cầu kinh doanh trong } \\
\text { tương lai của tôi trước khi tôi phân bồ nguồn lực của mình. }\end{array}$ \\
\hline \multicolumn{2}{|c|}{ Thang đo nhận thức về thành công (ACH-COG) } \\
\hline ACH-COG3 & $\begin{array}{l}\text { Để thành công, tôi tin rằng việc sử dụng thời gian của mình một cách khôn ngoan là } \\
\text { quan trọng. }\end{array}$ \\
\hline ACH-COG4 & $\begin{array}{l}\text { Tôi tin rằng để thành công một doanh nhân phải dành thời gian lập kế hoạch tương lai } \\
\text { cho công việc kinh doanh của mình. }\end{array}$ \\
\hline ACH-COG5 & $\begin{array}{l}\text { Tôi tin rằng một trong những chìa khóa để thành công trong kinh doanh là không trì } \\
\text { hoãn. }\end{array}$ \\
\hline ACH-COG6 & $\begin{array}{l}\text { Tôi tin rằng để thành công trong kinh doanh ngày nay, bạn phải loại bỏ sự thiếu hiệu } \\
\text { quả. }\end{array}$ \\
\hline ACH-COG7 & $\begin{array}{l}\text { Tôi tin rằng việc suy nghĩ về những khả năng trong tương lai quan trọng hơn là nghĩ về } \\
\text { những thành tích trong quá khứ. }\end{array}$ \\
\hline ACH-COG8 & $\begin{array}{l}\text { Tôi tin rằng điều quan trọng nhất trong việc lựa chọn đối tác kinh doanh là năng lực của } \\
\text { họ. }\end{array}$ \\
\hline \multicolumn{2}{|c|}{ Thang đo cảm xúc sáng tạo (INN-AFF) } \\
\hline INN-AFF3 & Tôi thích sử dụng các khái niệm \\
\hline INN-AFF7 & $\begin{array}{l}\text { Tôi thấy thực sự phấn chấn khi tôi nghĩ về những ý tưởng mới để thúc đẩy hoạt động kinh } \\
\text { doanh của tôi. }\end{array}$ \\
\hline INN-AFF8 & h là chất xúc tác cho sự thay đổi trong công việc kinh doanh. \\
\hline INN-AFF9 & Tôi thích thú khi làm những việc mới và \\
\hline \multicolumn{2}{|c|}{ Thang đo hành vi sáng tạo (INN-BEH) } \\
\hline & Tôi ít khi làm theo hướng dẫn, $\mathrm{t}$ \\
\hline & \\
\hline $\mathrm{H} 3$ & Tôi t \\
\hline$\overline{\mathrm{EH} 4}$ & Tôi t \\
\hline T-BEH5 & c kinh doanh theo những cách độc đáo. \\
\hline INN-BEH6 & $\begin{array}{l}\text { Tôi thường tìm kiếm những người đồng nghiệp thích khám phá những cách thức mới để } \\
\text { làm việc. }\end{array}$ \\
\hline \multicolumn{2}{|c|}{ Thang đo nhận thức sáng tạo (INN-COG) } \\
\hline INN-COG1 & $\begin{array}{l}\text { Tôi tin rằng những tổ chức không trải qua các thay đổi cơ bản bây giờ hay sau này sẽ có } \\
\text { xu hướng lâm vào bế tắc. }\end{array}$ \\
\hline INN-COG2 & $\begin{array}{l}\text { Tôi tin rằng để thành công, người ta phải tuân theo những tập quán kinh doanh được chấp } \\
\text { nhận rộng rãi. }\end{array}$ \\
\hline INN-COG3 & $\begin{array}{l}\text { Tôi tin rằng để thành công trong kinh doanh, bạn phải dành một khoảng thời gian mỗi } \\
\text { ngày để phát triển các cơ hội mới. }\end{array}$ \\
\hline INN-COG4 & $\begin{array}{l}\text { Tôi tin rằng để đi đến một giải pháp tốt cho một vấn đề kinh doanh, điều quan trọng là đặt } \\
\text { câu hỏi về những giả định được dùng đề xác định vấn đề. }\end{array}$ \\
\hline INN-COG5 & Tôi tin rằng việc tiếp tục tìm kiếm những cách thức mới trong kinh doanh là quan trọng. \\
\hline INN-COG6 & $\begin{array}{l}\text { Tôi tin rằng khi theo đuổi các mục tiêu kinh doanh thì kêt quả cuối cùng là quan trọng } \\
\text { hơn nhiều so với quá trình thực hiện theo các tập quán kinh doanh. }\end{array}$ \\
\hline INN-COG9 & oanh theo những cách độc đáo là quan trọng. \\
\hline
\end{tabular}

